\section{Persistent Paraplegia after Repeated Cardiac Arrests}

\section{British Medical fournal, 1969, 3, 572-573}

Resuscitation from cardiac arrest is an accepted procedure in hospital practice, but on occasion the appearance of signs of cerebral damage has led to the abandonment of efforts to revive such patients, and those recovering from circulatory arrest may have suffered some degree of irreversible brain damage (Jude et al., 1961). The cerebral hemispheres are the most vulnerable part of the central nervous system when ischaemia results from circulatory arrest (Brierley, 1962). The following case is recorded because the spinal cord appeared to have been selectively and irreversibly damaged by ischaemia after repeated cardiac arrest.

\section{CASE History}

A 63-year-old heating engineer was admitted to hospital on 12 March 1968 with a history of several hours' severe coronary pain during the preceding night. He showed no evidence of shock or cardiac failure. The pulse rate was $52 /$ minute and the blood pressure $150 / 80 \mathrm{~mm}$. Hg. At the apex a third heart sound was heard and the first sound was reduced. His nervous system was normal. An electrocardiogram (E.C.G.) showed anterior myocardial infarction, and the serum aspartate aminotransferase was 87 sigma-Frankel units $/ 100 \mathrm{ml}$. rising to 216 units on the second day. The Wassermann reaction was negative. Intravenous heparin was given and anticoagulant therapy was subsequently maintained with phenindione.

On 15 March there was transient pericardial and pleuropericardial friction associated with brief left pectoral discomfort and bilateral basal rales. His condition was then maintained until 26 March (14 days after admission), when ventricular fibrillation occurred and the standard emergency measures were applied without delay. After two D.C. shocks ventricular fibrillation was followed by asystole, and a further ventricular fibrillation occurred after injection of isoprenaline and calcium. Nine more D.C. shocks and intravenous lignocaine $(250 \mathrm{mg}$.) restored sinus rhythm, and the subsequent E.C.G. showed little change from its predecessor. The blood pressure was 100/40.

On 27 March he had a further cardiac arrest, which was quickly treated by external cardiac massage and D.C. conversion with resultant sinus rhythm. Some rib fractures occurred with paradoxical sternal movement, and many moist sounds were heard in the chest. He was treated with intravenous frusemide and intubation was performed with suction of much pus. Subsequently he was ventilated with 2 litres of oxygen and 9 litres of air per minute. The course of the treatment in relation to the cardiac arrests and

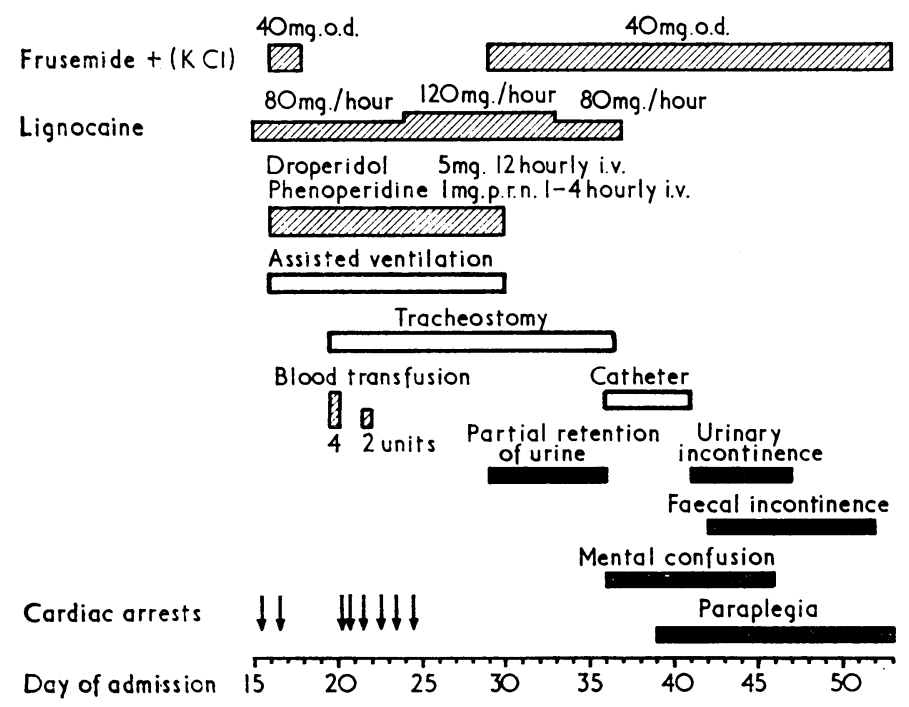

FIG. 1.-Clinical course and treatment. clinical progress is shown in Fig. 1. Antibiotics were given to prevent or treat chest infection during the four weeks after the first episode of cardiac arrest.

His condition was fairly satisfactory for the next 48 hours, but on 30 March after tracheostomy for insertion of a cuffed tube he bled profusely, and the tube had to be replaced after ligation of bleeding vessels. The prothrombin time was 45 seconds (control 14 seconds) and the phenindione dose was reduced. The haemoglobin level was 9.5 g. $/ 100 \mathrm{ml}$., and 4 pints $(2.3$ litres) of blood was administered over 16 hours. On 31 March he had two brief attacks of ventricular fibrillation, each responding to electrical defibrillation. The blood pressure was $100 / 60$ and the haemoglobin $9.6 \mathrm{~g} . / 100 \mathrm{ml}$. He was given a further 2 pints. $(1,140 \mathrm{ml}$.) of blood the next day.

Further attacks of ventricular fibrillation occurred on $1,2,3$, and 4 April and each responded quickly to defibrillation, though on two occasions this treatment had to be followed by cardiac massage (for asystole). After the last attack (23 days after admission) the rate of lignocaine administration was increased to $120 \mathrm{mg}$. per hour, At no point during the series of arrests was his circulation left unsupported for more than a minute, and there was no permanent loss of any peripheral pulse. Partial retention of urine was noted on 9 April and diarrhoea on 10 April. Two days after assisted respiration was ended (12 April) right basal pneumonic signs were detected. Urinary retention was complete on 16 April, and catheter drainage was instituted.

On being able to talk with the patient after the cuffed tracheostomy tube had been removed it became obvious that his cerebration was very slow; at times he was confused and his speech was hesitant. On 19 April the lower limbs were found to be almost powerless; there was minimal movement at the ankles and toes, and all lower limb reflexes (both deep and superficial) were absent. Sensation was evidently very defective in the lower limbs, but there also appeared to be transitory sensory loss in the hands and arms. On 22 April he was still mentally slow but less confused. There was analgesia of the lower limbs and trunk to about the level of D 8. After removal of the catheter he was incontinent of urine. Radiography of the thoracic and lumbar spine disclosed no abnormality.

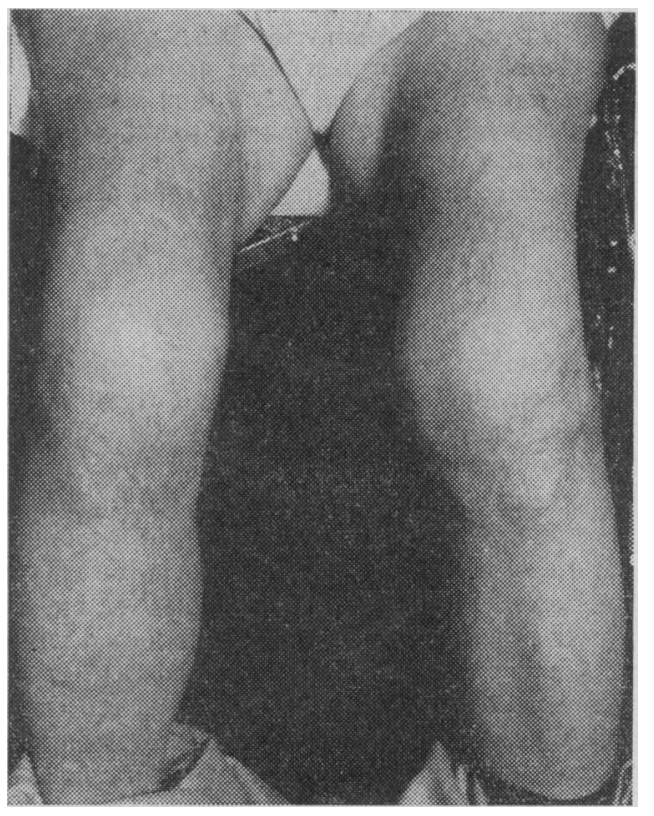

FIG. 2.-Quadriceps wasting (21 July 1968).

Urinary incontinence improved within a week, but faecal incontinence with diarrhoea took two weeks longer to make fuH recovery. Cerebration gradually improved to normal at the same time, and speech became quite clear. Nevertheless, his lower trunk remained so weak that he could not sit up unaided, and there was still marked weakness and flacoidity of the lower limbs with wasting of the quadriceps (Fig. 2). After the first few days there was minimal flexion power in the hips (stronger on the left), but this was insufficient to lift the limb against gravity. Hip extension seemed normal. Extension and flexion of the knees were very 
weak, slightly better on the left. Plantar flexion was good, but dorsiflexion was impaired, more in the ankles than the toes. Loss of sensation to pain and temperature persisted, a sensory level being established at D 8 on the left and D 10 on the right. There was normal appreciation of light touch, and joint position sensation was present in the toes. Vibration sense was absent in the lower limbs, though a few weeks later it had returned at all levels except the ankles. The ankle jerks were by then easily obtained, but the knee jerks remained diminished.

During the four months following the onset of the paraplegia he received anticoagulant and diuretic therapy, and his cardiac function continued to improve. There was a slight left ventricular heave and a slurred apical first sound; the blood pressure was 130/ 70 and the lungs were clear. Radiography showed that cardiac size had decreased since $23 \mathrm{March}$. He performed quite strenuous limb exercises without loss of breath or angina, but there was little further change in his neurological findings. He was discharged on 30 August 1968, walking quite well with leg callipers and the help of a Zimmer frame.

When seen again on 12 October, six months after the onset of paraplegia, there had been a slight generalized improvement in sensation to pain and temperature, though there was still a dorsal sensory level and an area of complete loss over the left shin. He was able to accomplish a slightly wider range of movements with his legs through skilful use of the available muscle power. It was doubtful whether the affected movements showed any true increase in power. Wasting of the quadriceps was still pronounced, and there had been no further change in the reflexes. Although all knee movements were weak, by hyperextending these joints he was able to stand unsupported and to walk while pushing his wheelchair. Using callipers he could walk 100 yards $(91 \mathrm{~m}$.). There was no heart failure and no cardiac enlargement clinically. Heart sounds were normal, and blood pressure was 155/80. The E.C.G. was unchanged.

\section{Comment}

From the clinical nature of the paraplegia with sensory loss and the lack of permanent cerebral damage it would seem that a defective blood supply to the spinal cord (possibly related to atheroma of the aorta) was a factor in the selective infarction of the cord. The main lesion was in the lower cord, where there was evidence of anterior horn damage at L 3 and L 4, and sensory levels were established at D 8 on the left and $\mathrm{D} 10$ on the right. The sensory defect was of the dissociated type, with marked loss of pain and temperature sensation and sparing of light touch and joint position modalities. Impairment of vibration sense was transient.

Henson and Parsons (1967) reported the case of a 59-year-old woman who developed an areflexic paraplegia and a low thoracic level of analgesia after a myocardial infarct which led to a 65minute episode of ventricular fibrillation. They suggested that cord infarction was precipitated by this temporary circulatory impairment, but that this may have been preconditioned by vascular damage in an earlier thoracoplasty for tuberculosis. In their case, as in ours, it seems reasonable to postulate that in the presence of local vascular disease an episode of hypotension was capable of producing cord infarction.

G. H. JenNings, M.D., F.R.C.P., Consultant Physician, Edgware General Hospital, Edgware, Middlesex.

M. A. NewTON, M.D., M.R.C.P., Consultant Physician, Central Middlesex Hospital ; formerly Senior Registrar in Medicine, Edgware General Hospital, Edgware, Middlesex.

\section{REFERENCES}

Brierley, J. B. (1962). Proceedings of the Royal Society of Medicine, 55, 659.

Henson, R. A., and Parsons, M. (1967). Quarterly fournal of Medicine, 36, 205.

Jude, J. R.; Kouwenhoven, W. B., and Knickerbocker, G. G. (1961). fournal of the American Medical Association, 178, 1063.

\section{Rupture of Choledochus Cyst in Pregnancy}

British Medical fournal, 1969, 3, 573-574

Since Vater first described the condition in 1723 many cases of choledochus cyst have been recorded in the literature. The subject was fully reviewed by Shallow et al. (1946) and Attar and Obeid (1955). Rupture of the cyst is a rare complication, only six cases having been documented by 1956 (Tagart, 1956); one of these, according to Friend (1958), occurred two weeks after a normal delivery.

A search of the literature confirms this case to be the first recorded instance of rupture of a choledochus cyst during pregnancy.

\section{CASE REPORT}

The patient, an unmarried primigravida aged 17, was first seen at the booking clinic on 7 June 1968. She gave a 10-year history of recurrent attacks of abdominal pain for which no cause had been found. In 1961 she had been admitted to hospital with a short episode of jaundice. The serum bilirubin level was $1.1 \mathrm{mg} . / 100 \mathrm{ml}$. A cholecystogram at that time showed poor concentration of the dye in the gall bladder, but no other abnormality. A diagnosis of infectious hepatitis was made. The jaundice faded and she was discharged after three weeks.

The pregnancy proceeded uneventfully until the twenty-eighth week, when she was admitted for investigation of severe epigastric pain radiating to the back. She had not vomited. There was no bowel or micturition upset and no vaginal bleeding.

On examination the patient was distressed and flushed, but did not appear ill ; her temperature was $98.2^{\circ} \mathrm{F}$. $\left(36.8^{\circ} \mathrm{C}\right.$.), pulse 80 per minute, and blood pressure $110 / 70 \mathrm{~mm}$. Hg. The abdomen was soft, and there was some tenderness in the epigastrium and in the right costal margin. Full blood counts, electrolytes, urea, and serum amylase were normal. The urine was not infected and did not contain porphyrins.

Twelve hours after admission the patient experienced sudden knife-like pains in the right iliac fossa; these had gradually spread to involve the whole abdomen. Her symptoms were unlike those of previous attacks and were accompanied by profuse vomiting. Her temperature was $100^{\circ} \mathrm{F}$. $\left(37 \cdot 8^{\circ} \mathrm{C}\right.$.) and pulse 110 . There was a strong fetor oris. The abdomen was rigid to palpation with maximal guarding in the right iliac fossa and pronounced rebound tenderness. A diagnosis of perforated acute appendicitis was made and laparotomy was performed on 20 October.

Operative Findings.-The peritoneal cavity contained a large quantity of heavily bile-stained fluid. Retroperitoneal oedema and bile staining were present in the region of the head of the pancreas, extending up into the lesser omentum and down along the right paracolic gutter. The gall bladder was collapsed, with no stones. The pancreas could not be identified with certainty on account of the oedema. The lesser sac was obliterated by adhesions and the aditus could not be seen. Though there was no evidence of fat necrosis a tentative diagnosis of acute oedematous pancreatitis was made. Cholecystostomy was performed and the abdomen was closed, leaving a drain to the region of the pancreas.

The patient was transferred to the intensive care unit and went into labour 24 hours later. Delivery was by low forceps under pudendal block. The infant, a girl of $3 \mathrm{lb} .9 \mathrm{oz}$. (1,600 g.), at first 